\title{
Polarised cross-sections in DIS at HERA
}

\section{Hiroshi Kaji}

on behalf of the H1 and ZEUS collaborations

Tokyo Metropolitan University, Japan

E-mail: kaji-hiroshi@phys.metro-u.ac.jp

Cross sections for charged and neutral current deep inelastic scattering in collisions between protons and longitudinally polarised electron or positron beams at a centre-of-mass energy of 318 $\mathrm{GeV}$ have been measured. The cross section for the charged current process at $Q^{2}>400 \mathrm{GeV}^{2}$ and $y<0.9$ was measured with the two different beam polarisations. It clearly shows the lefthanded nature of the weak interaction. By extrapolating the measurements of the H1 and ZEUS experiments to the fully polarised states, the right-handed charged current cross section is measured to be $\sigma_{R H C C}^{e^{+} p}=0.2 \pm 1.8$ (stat.) \pm 1.6 (sys.) pb (prelim.), consistent with zero as the Standard Model predicts.

International Europhysics Conference on High Energy Physics

July 21st - 27th 2005

Lisboa, Portugal 


\section{Introduction}

Deep Inelastic Scattering (DIS) is a key tool for understanding the structure of the proton. The HERA $e^{ \pm} p$ collider provides DIS events in the large negative four-momentum-transfer squared, $Q^{2}$, region. Two kinds of reactions are involved in DIS. The neutral current (NC) reaction, which is mediated by a $\gamma$ or $Z^{0}$ boson. The other is the charged current (CC) reaction, which is mediated by the charged $W^{ \pm}$boson. At HERA's large $Q^{2}$ region, both the CC and $\mathrm{NC}$ weak interactions in $e^{ \pm} p$ scattering can be measured. The $\mathrm{H} 1$ and ZEUS collaborations have reported measurements of unpolarised cross sections for CC and NC DIS [1] using the data collected in the years 1994-2000 (HERA-I).

Since 2002, the second phase of HERA operation (HERA-II) has started to provide higher luminosity and longitudinally polarised $e^{ \pm}$beams to the collider experiments. The polarisation of the $e^{ \pm}$beam $(\mathrm{P})$ is defined as $\left(N_{R}-N_{L}\right) /\left(N_{R}+N_{L}\right)$, where $N_{R}$ and $N_{L}$ are the number of the right-handed and left-handed $e^{ \pm}$in the beam, respectively. Since the weak interaction is purely left-handed, the cross sections varies as a function of $P$. The measurements at HERA give a direct approach to the right-handed weak interaction.

This paper presents the measurements of the cross sections for CC and NC DIS using longitudinally polarised $e^{ \pm}$beams and unpolarised protons [2][3]. The measured cross section are compared to the Standard Model (SM) predictions. The data were recorded in the years 2003-2005 at a centre-of-mass energy of $318 \mathrm{GeV}$. The ZEUS integrated luminosity is 14.1 (16.4) $\mathrm{pb}^{-1}$ with $\mathrm{P}$ of $+0.318(-0.402)$ for $e^{+} p$ scattering and $8.3(45.1) \mathrm{pb}^{-1}$ with $\mathrm{P}$ of $+0.292(-0.259)$ for $e^{-} p$ scattering. The $\mathrm{H} 1$ integrated luminosity is $15.3(21.7) \mathrm{pb}^{-1}$ with $\mathrm{P}$ of $+0.330(-0.402)$ for $e^{+} p$ scattering and $17.8 \mathrm{pb}^{-1}$ with $\mathrm{P}$ of -0.254 for $e^{-} p$ scattering.

\section{Charged current analysis}

The kinematics of inclusive DIS can be defined by the three variables; $Q^{2}$, Bjorken $x$, and the inelasticity, $y$. They are related by $Q^{2}=s x y$, where $s$ is the square of the centre-of-mass energy (neglecting the masses of the incoming particles). The cross section is, therefore, described by a pair of these three variables. The polarised CC cross section is given by

$$
\frac{d^{2} \sigma_{p o l C C}^{e^{ \pm} p}}{d Q^{2} d x}=\frac{(1 \mp \mathrm{P})}{2} \cdot \frac{d^{2} \sigma_{R H C C}^{e^{ \pm} p}}{d Q^{2} d x}+\frac{(1 \pm \mathrm{P})}{2} \cdot \frac{d^{2} \sigma_{L H C C}^{e^{ \pm} p}}{d Q^{2} d x},
$$

where $\sigma_{R H C C}^{e^{ \pm} p}$ and $\sigma_{L H C C}^{e^{ \pm} p}$ denote the CC DIS cross sections with right-handed and left-handed $e^{ \pm}$ beams, respectively. Since the CC interaction is a purely weak process, $\sigma_{R H C C}^{e^{ \pm} p}=0$ in the SM.

Figure 1 shows the single differential cross sections in $Q^{2}, x$, and $y$ for $e^{-} p$ scattering measured by the ZEUS experiment. The lines in each plot show the polarised cross sections predicted by the SM. The cross section changes according to the polarisation in the same way in each measured kinematic variable and is consistent with the SM. Figure 2 is the cross sections for $Q^{2}>400 \mathrm{GeV}^{2}$ and $y<0.9$. The cross sections measured from both H1 and ZEUS are plotted together with the results of the unpolarised beams. The lines represent the polarisation dependence from the SM prediction. The right handed CC cross section determined from the combined $\mathrm{H} 1$ and ZEUS results 

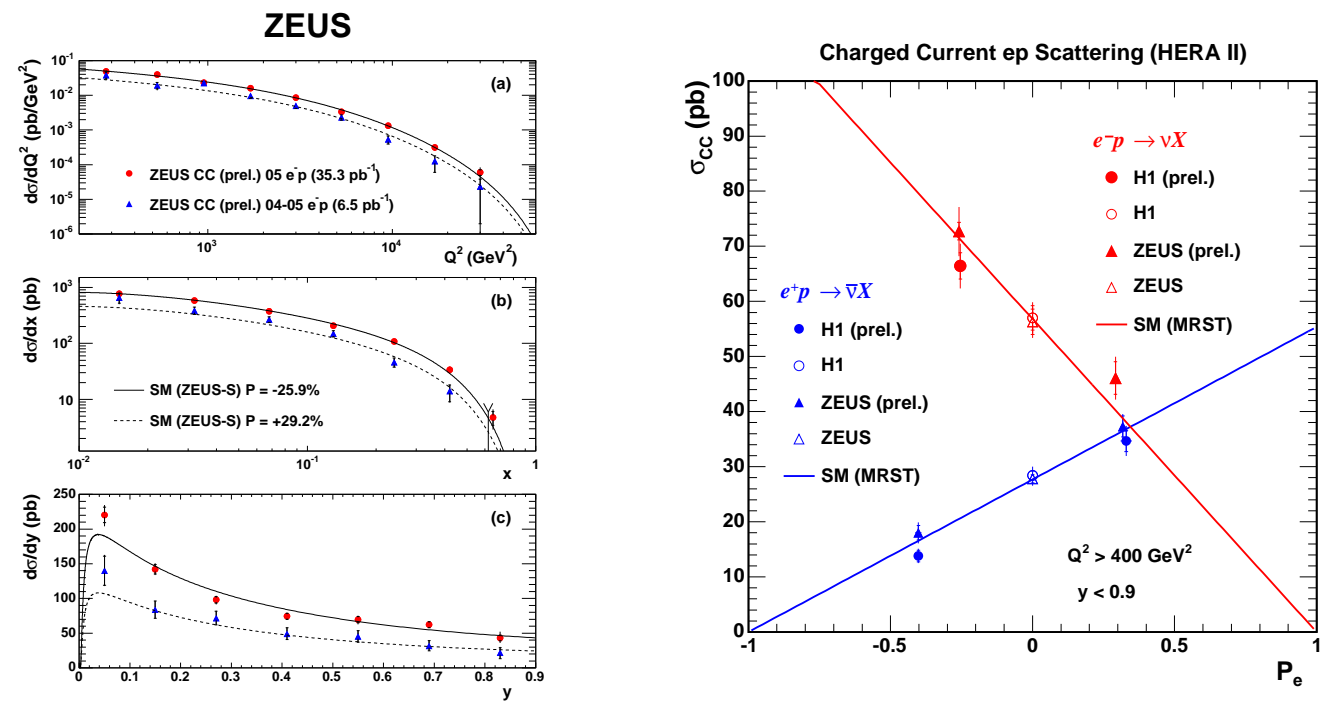

Figure 1: The single differential cross sections for Figure 2: The cross section at $Q^{2}>400 \mathrm{GeV}^{2}$ and $e^{-} p$ measured by ZEUS. $y<0.9$ measured by $\mathrm{H} 1$ and ZEUS.

of $e^{+} p$ scattering is preliminarily extrapolated to give,

$$
\sigma_{R H C C}^{e^{+} p}=0.2 \pm 1.8(\text { stat. }) \pm 1.6(\text { sys. }) \text { pb. }
$$

The result suggests the absence of right-handed currents, as consistent with the SM.

\section{Neutral current analysis}

The polarised $\mathrm{NC}$ cross section is given by

$$
\frac{d^{2} \sigma_{N C}^{e^{ \pm}(p o l) p}}{d Q^{2} d x}=\frac{2 \pi \alpha^{2}}{x Q^{4}}\left[H_{0}^{ \pm}+\mathrm{P} H_{\mathrm{P}}^{ \pm}\right]
$$

where $\alpha$ is the fine structure constant and $H_{0}^{ \pm}$and $H_{\mathrm{P}}^{ \pm}$contain the unpolarised and polarised structure functions, respectively. In contrast to the $\mathrm{CC}$, the polarisation dependence is seen only in the $Q^{2}$ region where the $Z^{0}$ contribution plays a significant role. Therefore, $H_{\mathrm{P}}^{ \pm}$is visible only at very large $Q^{2}$.

The kinematic distributions of $\mathrm{NC}$ events measured by $\mathrm{H} 1$ are plotted in Figure 3. We can conclude that the experimental conditions are well undercontrolled. Figure 4 (a) and (b) show the single differential cross sections for $e^{-} p$ scattering measured by ZEUS, as a function of $Q^{2}$. In order to make the polarisation effect visible, the ratio between the two cross sections is plotted in Figure 4 (c). The SM prediction shown as the dotted line indicates a subtle asymmetry of the cross section at large $Q^{2}$. The data look to show the polarisation effect, however, the present precision does not allow a definite conclusion to be drawn. 

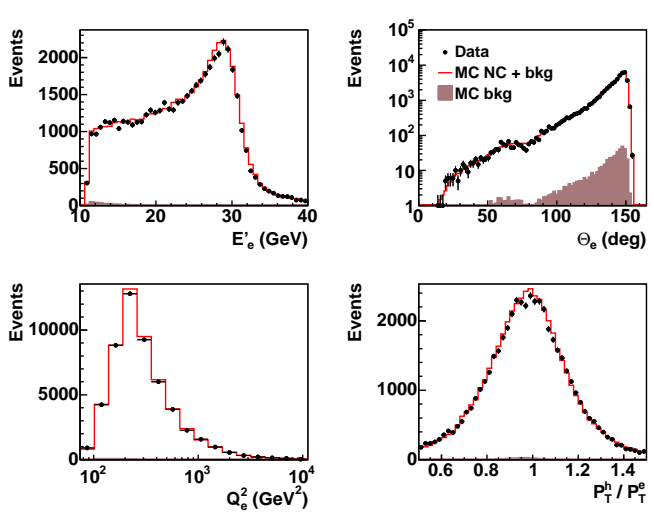

Figure 3: The kinematic distribution of $\mathrm{NC}$ events measured by $\mathrm{H} 1$.
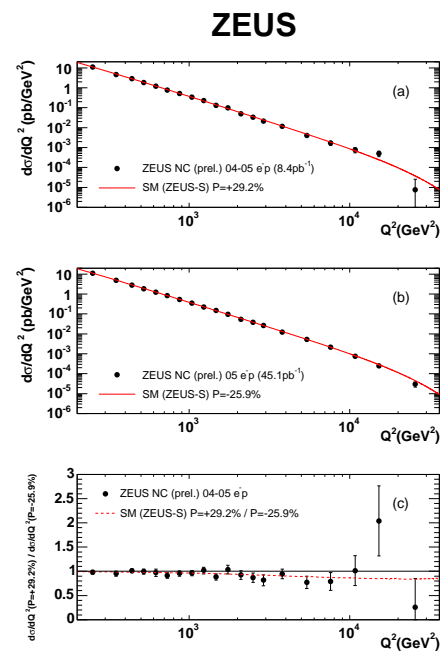

Figure 4: The NC cross section, $d \sigma / d Q^{2}$ for $e^{-} p$ measured by ZEUS.

\section{Summary}

The measured cross sections for CC and NC DIS with longitudinally polarised $e^{ \pm} p$ beams, were presented. For the $\mathrm{CC}$ process, the polarisation dependence of the single differential cross sections were observed in all measured kinematic regions. The cross sections at $Q^{2}>400 \mathrm{GeV}^{2}$ and $y<0.9$ were measured for both $e^{ \pm} p$ scattering. The right-handed CC cross section was derived by linear extrapolation to $P=-1$ with the combined H1 and ZEUS $e^{+} p$ scattering results. It is consistent with zero and the SM, where the right-handed weak interaction is absent.

The single differential NC cross sections as a function of $Q^{2}$ were also measured. The effect of the polarisation has the same trend as the SM prediction, however, the present precision does not allow a definite conclusion to be drawn.

\section{References}

[1] for example, H1 Coll., C. Adloff et al., Eur. Phys. J. C 30, 1 (2003).

ZEUS Coll., S. Chekanov et al., Eur. Phys. J. C 32, 1 (2003).

ZEUS Coll., S. Chekanov et al., Phys. Rev. D 70, 052001 (2004).

[2] H1 Coll., Measurement of the Polarisation Dependence of the Total $e^{+} p$ Charged Current Cross Section., Abstract 620, submitted to the HEP2005 conference on July 21-27, 2005.

H1 Coll., Measurement of the Polarisation Dependence of the Total $e^{-} p$ Charged Current Cross Section., Abstract 621, submitted to the HEP2005 conference on July 21-27, 2005.

[3] ZEUS Coll., Measurement of high- $Q^{2}$ deep inelastic scattering cross sections with longitudinally polarised positron beams at HERA.,

Abstract 831, submitted to the HEP2005 conference on July 21-27, 2005.

ZEUS Coll., Measurement of high- $Q^{2}$ deep inelastic scattering cross sections with longitudinally polarised electron beams at HERA.,

Abstract 325, submitted to the HEP2005 conference on July 21-27, 2005. 